\section{Evaluating the use of microscopic examination and rapid diagnostic tests to diagnose malaria in North Central Nigeria}

\author{
Innocent C. Omalu, ${ }^{1}$ George Uzoaga, ${ }^{1}$ \\ Israel Kayode Olayemi, ${ }^{1}$ \\ Charles Mgbemena, ${ }^{2}$ Suleiman Hassan, ${ }^{3}$ \\ Victoria Pam, ${ }^{4}$ Adeniran Lateef, ${ }^{5}$ \\ Samuel Sunday Eke ${ }^{1}$ \\ 'Department of Biological Sciences, \\ Federal University of Technology, Minna; \\ ${ }^{2}$ Dentistry Department, Niger State \\ General Hospital, Minna; ${ }^{3} \mathrm{ABT}$ \\ Development Foundation - PMI-ARS \\ Project, Lafia; ${ }^{4}$ National Veterinary \\ Research Institute, Vom; ${ }^{5}$ Department \\ of Physiology and Biochemistry, \\ University of Abuja, Nigeria
}

\section{Abstract}

The global impact of malaria has spurred interest in developing prompt and accurate diagnostic strategies to provide an effective management of the disease. The aim of this study was to compare rapid diagnostic tests (RDTs) for malaria with routine microscopy. Samples were collected randomly from 364 febrile out-patients with clinical suspicion of malaria from four hospitals in North Central Nigeria. Results from the rapid diagnostic kits were analysed and compared to those obtained by general microscopy. Of the 364 out-patients involved in the study, 218 (59.89\%) tested positive for Plasmodium falciparum by RDTs, whereas 263 (72.256\%) tested positive by microscopy. There are significant differences $(\mathrm{P}<0.05)$ in infection rates between RDT and microscopy. The sensitivity, specificity and negative predictive values of RDTs compared to microscopy are low, while the positive predictive value is high. Evaluation of RDTs against the parasite-positive panel with parasite densities of $<1000$ parasites/ $\mu \mathrm{L}$, between 1000-5000 parasites/ $\mu \mathrm{L}$ and above 5000 parasites/ $\mu \mathrm{L}$ was $11.73,30.61,57.65 \%$ for RDTs compared to $6.11,27.95$ and $65.94 \%$ for microscopy, respectively. Test line intensity increases with increase in parasite densities for both methods.

\section{Introduction}

Malaria is the most common single diagnosis made in most countries in Africa. ${ }^{1}$ The accuracy of clinical diagnosis is limited by the low specificity of symptoms and signs of malaria. ${ }^{2}$ Presumptive anti-malaria treatment for any fever without scientific investigation of possible alternative causes is widely practiced, and studies suggest that this leads to significant over-use of anti-malarial medicines throughout Africa. ${ }^{3,4}$ With growth of resistance to older anti-malarial drugs, newer but more expensive drugs have been developed as first line drugs, like the artemisinin combination therapy which has largely replaced the older drugs like Chloroquine and Sulphadoxine/ Pyrimethamine in most African countries. ${ }^{5}$ The widespread availability and affordability of these drugs especially in rural areas depend on subsidy. ${ }^{6}$ This may become unsustainable if most anti-malarial drugs continue to be given to patients who do not have malaria. If patients with bacterial disease, an important cause of avoidable death in children in Africa ${ }^{7,8}$ are treated as malaria cases, they may not receive appropriate treatment.

The World Health Organization has recommended that management of all malaria cases should be confirmed by quality-assured, parasite-based diagnosis before treatment is started. ${ }^{10,11}$ Improving the diagnosis of acute febrile illness so that anti-malaria drugs are targeted to patients who need them and alternative diagnosis sought in others is therefore a matter of public health priority in Africa.

In Nigeria one of the four key interventions towards malaria control is the introduction of parasitological confirmation of malaria cases by rapid diagnostic tests (RDTs) and scaling up of diagnosis by microscopy. ${ }^{12}$ The general objective being to achieve timely and equitable access to malaria diagnosis and treatment by all sections of the population and as close to the home as possible. The use of RDTs as a definitive diagnostic tool where microscopy is not readily available to confirm suspected malaria cases have been proposed; however, its performance must be evaluated to avoid over or under diagnosis of malaria cases before it can replace a more tedious microscopy. The specific objective of the study is to examine performance of RDTs compared to microscopy which is the gold standard in North Central Nigeria.

\section{Materials and Methods}

\section{Study area}

The study was carried out in hospital settings. Four hospitals with large attendance and standard diagnostic laboratories were selected, namely Adonai Hospital Mararaba, Nasarawa State, Blessed Trinity Hospital in New Karu Local Government, Jikwoyi Medical Centre in
Correspondence: Innocent Chukwuemeka Omalu, Department of Biological Sciences, Federal University of Technology, Zungeru Road, 920211 Minna, Nigeria.

Tel: +234.8053.454705.

E-mail: omaluicj@futminna.edu.ng

Key words: malaria, RDTs, microscopy, diagnosis, out-patients.

Conflict of interests: the authors declare no potential conflict of interests.

Acknowledgments: the authors gratefully acknowledge the assistance of the Laboratory technologists of Adonai Hospital Mararaba and Blessed Trinity Hospital in New Karu in Nasarawa State, Jikwoyi Medical Centre and Asokoro District Hospital in Abuja, Nigeria.

Received for publication: 22 July 2013.

Revision received: 30 June 2014.

Accepted for publication: 30 June 2014.

This work is licensed under a Creative Commons Attribution NonCommercial 3.0 License (CC BYNC 3.0).

(C) Copyright I.C. Omalu et al., 2014

Licensee PAGEPress, Italy

Malaria Reports 2014; 3:1836

doi:10.4081/malaria.2014.1836

Abuja Municipal Area Council and Asokoro District Hospital, with Hospital Attendance cutting across the Abuja, Kogi and Niger and Nasarawa States as well as other parts of the North Central Zone. The study was conducted over a period of 5 months from February to June, part of which fell in the rainy season when the prevalence of malaria is normally at its peak.

\section{Study population}

From among febrile hospital attendees [patients with fever $\left(\geq 37.5^{\circ} \mathrm{C}\right), 364$ patients (91 per hospital] were selected. Test outcomes were recorded for each of the centres. Positive cases were further examined to ascertain par-

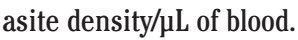

\section{Parasitological analysis}

Thick and thin smears were made on the same slide for each patient from finger prick using a sterile lancet. Two slides were made for each patient. The first slide was the read (R) slide (that is the slide that was read), while the other slide was archived (A) slide. The thin films were fixed with methanol and left to dry. The prepared smears were stained with $10 \%$ Giemsa at a pH of 7.2 for $35 \mathrm{~min}$. The staining process was quality controlled to ensure that the morphology of the malaria parasites in positive slides were distinct and clear. The parasite den- 
sity was computed as the number of parasites per 500 leucocytes on a thick film and reported as parasites per microlitre of blood assuming an average white blood-cell count of 8000 per $\mu L^{13}$ Stained slides were examined under the light microscope using $\times 100$ objective lens (immersion oil). A slide was considered negative after 100 high power fields have been examined. Another Microscopist, the second reader, was made to re-read each slide. Parasite counts of $>20 \%$ discordance between two readers were re-read by a third reader, who served as the tie breaker.

\section{Rapid diagnostic tests}

This devise detected malaria antigen in a small amount of blood, usually 5-15 $\mu \mathrm{L}$, by immune-chromatographic assay with monoclonal antibodies directed against the target parasite agent (Plasmodium falciparum and Plasmodium vivax) and impregnated on a test strip. The results usually a coloured test line was obtained after 5-20 min. RDT requires no capital investment or electricity, are simple to perform and easy to interpret. Sensitivity and specificity tests were performed on 2 RDTs namely First Response (Premier Medical Corporation, Watchung, NJ, USA) and CTK (Biotech Inc., Oklahoma City, OK, USA). CTK was used in this study because it was more sensitive and specific.

\section{Ethical approval}

Ethical approval was obtained from the management of each of the participating hospitals including the ethics committees of Federal Capital of the Territory of Abuja, Nigeria.

\section{Data analysis}

The collected data were analysed using SPSS version. Proportions were compared by Chi-square tests.

\section{Results}

RDT showed detection of $P$. falciparum in 218 (59.89\%) out of 364 out-patients with males 103 (47.25\%) and females 115 (52.75\%) tested from the four hospitals; whereas microscopic results showed detection of of $P$. falciparum in $263(72.25 \%)$ out of 364 patients with male 118 (44.87\%) and females 145 (55.13\%) tested from the four hospitals (Table 1). Chisquare analysis showed that the increase in difference between the RDT and the microscopy results was significant $(\mathrm{P}<0.05)$.
The sensitivity, specificity, positive predictive value and negative predictive values of RDTs against blood slide microscopy for all species of malaria combined are shown in Table 2 . The overall sensitivity was $45.32 \%$ (95\% CI 3.92 5.84). Specificity of RDTs was $40.89 \%$ (3.42$5.01)$ overall. The overall positive predictive value was $59.89 \%$, while negative predictive value was very low (27.75\%).

Test performance showed that evaluation of RDTs against the parasite-positive panel with parasite densities of $<1000$ parasites $/ \mu \mathrm{L}$ revealed $11.73 \%$, between $1000-5000$ para-

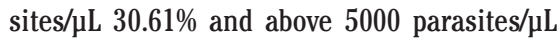
$57.65 \%$ compared to $6.11,27.95$ and $65.94 \%$ for microscopy, respectively (Table 3). Analysis showed that RDT is also sensitive $(\mathrm{P}>0.05)$ compared to microscopic examination.

\section{Discussion}

Recently in Nigeria the use of RDT as a fast and easier method of malaria diagnosis has been sponsored to replace the tedious and time-consuming microscopic methods. Still, this study was initiated to compare the sensitivity of RDTs in accurately determining malar-

Table 1. Detection of Plasmodium falciparum in peripheral blood of out-patients according to gender using rapid diagnostic test and microscopy.

\begin{tabular}{|c|c|c|c|c|c|c|}
\hline \multirow{2}{*}{ Categories } & \multicolumn{2}{|c|}{ Overall infected patients } & \multicolumn{2}{|c|}{ Male infected patients } & \multicolumn{2}{|c|}{ Female infected patients } \\
\hline & n & $\%$ & n & $\%$ & n & $\%$ \\
\hline RDT & 218 & 59.89 & 103 & 47.25 & 115 & 52.75 \\
\hline Microscopy & 263 & 72.25 & 118 & 44.87 & 145 & 55.13 \\
\hline
\end{tabular}

RDT, rapid diagnostic test.

Table 2. Performance of different rapid diagnostic methods compared to Giemsa stain microscopy.

\begin{tabular}{lcccccc}
\multicolumn{2}{c}{ Nicroscopy } & Sensitivity (\%) $(95 \%$ CI) & Specificity (\%) (95\% CI) & PPV (\%) \\
& Positive & Negative & & & NPV (\%) \\
RDTs & & & & $40.89(3.42-5.01)$ & 59.8 \\
Positive & 218 & 146 & $45.32(3.92-5.84)$ & - & - \\
Negative & 263 & 101 & - & - & - \\
\hline
\end{tabular}

RDTs, rapid diagnostic tests; PPV, positive predictive value; NPV, negative predictive value; CI, confidence interval.

Table 3. Estimation of parasite density of the thick film positive results $v$ s test line intensity of rapid diagnostic test positive results from four selected hospitals in North Central Nigeria.

\begin{tabular}{|c|c|c|c|c|c|c|}
\hline \multirow[t]{2}{*}{ Hospital } & \multicolumn{3}{|c|}{ Microscopy, parasite density $(\mu / L)$} & \multicolumn{3}{|c|}{ RDT, test line intensity } \\
\hline & $<1000 / \mathrm{UL}$ & 1000-5000/UL & $>5000 / \mathrm{UL}$ & Faint & Visible & Very visible \\
\hline Adonai Hospital Mararaba & 30 & 27 & 5 & 8 & 12 & 32 \\
\hline Blessed Trinity Hospital & 47 & 14 & 2 & 6 & 13 & 29 \\
\hline Jikwoyi Medical Centre & 45 & 11 & 3 & 4 & 17 & 25 \\
\hline Asokoro District Hospital & 29 & 12 & 4 & 5 & 18 & 27 \\
\hline \multicolumn{7}{|l|}{ Total } \\
\hline $\begin{array}{l}\mathrm{n} \\
\%\end{array}$ & $\begin{array}{c}151 \\
65.94\end{array}$ & $\begin{array}{c}64 \\
27.95\end{array}$ & $\begin{array}{c}14 \\
6.11\end{array}$ & $\begin{array}{c}23 \\
11.73\end{array}$ & $\begin{array}{c}60 \\
30.61\end{array}$ & $\begin{array}{c}113 \\
57.65\end{array}$ \\
\hline
\end{tabular}


ia infections. Results showed that prevalence of malaria is generally high in North Central Nigeria. Despite the difference between the overall prevalence of malaria detected by the two tests in our study, the overall sensitivity observed was low (45.32\%). This indicates that there was a substantial non-overlap between the positives detected by each test. The positive predictive value was generally high (60\%). Other reports indicate that RDTs have shown a comparable level of accuracy to microscopy in clinical settings. ${ }^{14,15}$ Even though the clinical history of the participants was not recorded in our study, evidence from other studies showed that RDT positive cases missed by microscopy might be individuals who had been treated but in whom antigenemia persists. ${ }^{15,16}$

Jamshaid and others in a similar study compared two commercial assays, RDTs with microscopy for confirmation of symptomatically diagnosed malaria where a total of 750 patients were examined. The ICT malaria Pf/Pv test kit failed to detect malaria infection in 93 (34\%) of 271 malaria patients and optimal test failed to detect malaria infection in 41 (15\%) of 271 malaria patients. The sensitivities of ICT malaria Pf/Pv and OptiMAL tests for detection of $P$. falciparum infection were 81 and $87 \%$ respectively and their sensitivities decreased significantly to 23 and $44 \%$ respectively at parasite densities of $<500 / \mu \mathrm{L}$ which is similar to the result obtained in this study. ${ }^{17}$

In another work on RDTs and microscopy for guiding outpatient treatment of febrile illness in Tanzania: a randomized trial, $49.22 \%$ tested positive by microscopy while $46.16 \%$ tested positive by RDT. The factor of parasite density in the sensitivity of RDT in detecting malaria parasite in peripheral blood played a vital role here $^{9}$ and other studies have shown that test line intensity increases with increase in parasite densities. ${ }^{18}$ The lack of sensitivity of RDTs at low parasitaemia compared to microscopy is one of the shortcomings noted here which is also reflected in our findings.

Moody and others pointed out that RDTs can be useful in screening febrile returnees from endemic areas ${ }^{19}$ and according to World Health Organization, on the use of RDTs, they are recommended in situations exceeding microscopy capability such as in an outbreak or in occupationally exposed groups..$^{20}$ As RDTs improve, including in sensitivity and in ability to measure parasitaemia levels, at least semi-quantitatively, the scope of RDT application will expand. Current RDTs cannot replace microscopy; other factors such as the quality of the products, storage temperature and humidity, and end users' performance can affect the diagnostic accuracy.

\section{Conclusions}

In conclusion, the results of this study adds to the evidence that non-microscopic rapid tests for the detection of plasmodial antigens may develop into important diagnostic tools and can prove to be a valuable adjunct to clinical assessment of the patient's blood film microscopy under certain circumstances. The study design was such as to make a direct comparison of the performance of microscopy and RDTs in four different hospitals. These tests are rapid and simpler to perform and to interpret, however, their sensitivity indicates that they should not yet be regarded as first line diagnostic tests, but with time, further improvement in technology of production and better storage and handling, they hold the promise of becoming the first line diagnostic tools for malaria taking over from microscopy which remains the gold standard for now.

\section{References}

1. WHO. The Africa malaria report 2003. Geneva, Switzerland: World Health Organization; 2003.

2. Kallander K, Nsungwa-Sabiiti J, Peterson S. Symptom overlap for malaria and pneumonia: policy implications for home management strategies. Acta Trop 2004;90: 211-4.

3. Makani J, Matuja W, Liyombo E, et al. Admission diagnosis of cerebral malaria in adults in an endemic area of tanzania: implications and clinical description. Q J Med 2003;96:355-62.

4. Zurovac D, Midia B, Ochola SA, et al. Microscopy and outpatient malaria management among older children and adults in Kenya. Trop Med Int Health 2006;11:432-40.

5. White NJ, Nosten F, Looareesuwan S, et al. Averting a malaria disaster. Lancet 1999;353:1965-7.

6. Global Fund. Global fund on malaria treatments: global fund 2006. Available from: http://www.theglobalfund.org/en/publications/annualreports/

7. Brent AJ, Ahmed U, Ndiritu M, et al. Incidence of clinically significant bacteriamia in children who present to hospital in Kenya: community based observational study. Lancet 2006;367:482-8.

8. WHO. The use of malaria rapid diagnostic tests. Geneva, Switzerland: World Health
Organization; 2006.

9. Reyburn H, Mbatia R, Drakeley G, et al. Over diagnosis of malaria in patients with severe febrile illness in Tanzania: a prospective study. Brit Med J 2004;329: 1212.

10. WHO. Malaria rapid diagnostic test performance; results of WHO product testing of malaria RDTs: round 2. Geneva, Switzerland: World Health Organization; 2009.

11. WHO. Parasitological confirmation of malaria diagnosis. Geneva, Switzerland: World Health Organization; 2009.

12. Federal Ministry of Health. A road map for malaria control in Nigeria: strategic plan. National Malaria Control Programme, Federal Ministry of Health, Abuja, Nigeria; 2008.

13. Greenwood BM, Armstrong JRM. Comparison of two simple methods for determining malaria parasite density. Trans R Soc Trop Med Hyg 1991;85:186-8.

14. Moonasar D, Goga AE, Frean J, et al. An exploratory study of factors that affect the performance and usage of rapid diagnostic tests for malaria in the Limpopo Province, South Africa. Malaria J 2007;6:74.

15. Moody A. Rapid diagnostic tests for malaria parasites. Clin Microbiol Rev 2002; 15:66-78.

16. WHO. The role of laboratory diagnosis to support malaria disease management: focus on the use of rapid diagnostic tests in areas of high transmission, report of a WHO technical consultation. Geneva, Switzerland: World Health Organization; 2008.

17. Jamshaid I, Nabila K. Comparism of two commercial assays with expert microscopy for confirmation of symptomatically diagnosed malaria. Am J Trop Med Hyg 2002; 60:109-18.

18. Forney JR, Magill, AJ, Wongsrichanalai C, et al. Malaria rapid diagnostic devices: performance characteristics of the parasight $\mathrm{F}$ device determined in a multisite filed study. J Clin Microbiol 2001;39:2884-90.

19. Moody AH, Chiodini P. Non microscopic method for malaria diagnosis using OptMAL IT, a second-generation dipstick for malaria pLDH antigen detection. Br J Biomed Sci 2002;59:228-31.

20. WHO. The use of malaria diagnostic tests. Manila, Philippines: World Health Organization Regional Office for the Western Pacific; 2004. 